\title{
EchoGéo
}

$44 \mid 2018$

Les valorisations territoriales et touristiques du street art

\section{Les métiers de la territorialisation du street art : de l'intermédiation à la valorisation}

Sébastien Jacquot, Olivier Landes, Elise Herszkowicz, JBC, François Roblot, Damien Cacouault and Thom Thom

\section{(2) OpenEdition}

Journals

Electronic version

URL: https://journals.openedition.org/echogeo/15533

DOI: $10.4000 /$ echogeo. 15533

ISSN: 1963-1197

Publisher

Pôle de recherche pour l'organisation et la diffusion de l'information géographique (CNRS UMR 8586)

Electronic reference

Sébastien Jacquot, Olivier Landes, Elise Herszkowicz, JBC, François Roblot, Damien Cacouault and Thom Thom, "Les métiers de la territorialisation du street art : de l'intermédiation à la valorisation", EchoGéo [Online], 44 | 2018, Online since 31 July 2018, connection on 31 July 2021. URL: http:// journals.openedition.org/echogeo/15533 ; DOI: https://doi.org/10.4000/echogeo.15533

This text was automatically generated on 31 July 2021.

EchoGéo est mis à disposition selon les termes de la licence Creative Commons Attribution - Pas d'Utilisation Commerciale - Pas de Modification 4.0 International (CC BY-NC-ND) 


\title{
Les métiers de la territorialisation du street art : de l'intermédiation à la valorisation
}

\author{
Sébastien Jacquot, Olivier Landes, Elise Herszkowicz, JBC, François \\ Roblot, Damien Cacouault and Thom Thom
}

\section{Introduction}

1 Le street art s'inscrit dans des configurations territoriales multiples, impliquant un grand nombre d'acteurs, au-delà des seuls artistes, faisant le lien avec les collectivités commanditaires et prescriptives, et/ou les publics du street art, des habitants aux visiteurs et touristes.

2 Ces acteurs proviennent d'institutions, d'associations variées, ancrées localement ou intervenant en intermédiaires sur des territoires multiples. Comment ces différentes provenances jouent sur les modes de valorisation de street art, les positionnements à l'égard de sa valorisation, les types de projet qui sont menés et les rapports aux usagers ? Quelles sont les compétences, façons de faire, relations nouées entre acteurs, autour du street art, bref comment se construisent des mondes de l'art (au sens d'H. Becker) qui sont aussi des mondes de la ville? En effet, l'enjeu de la fabrique urbaine apparaît structurant à plusieurs titres : à travers des formes d'institutionnalisation du street art se joue l'articulation entre street art et ville, dans son lien au renouvellement urbain, au marketing territorial, aux appropriations par des publics d'origine variée, à la valorisation touristique des espaces urbains au centre-ville et en périphéries, et qui peut concerner aussi bien des sites historiques que des lieux en régénération.

3 L'enjeu de la fabrique urbaine questionne aussi le fonctionnement des mondes de l'art, les conditions d'activités des artistes, l'évolution des formes, des objectifs et des publics des créations.

4 En ce sens, ces enjeux questionnent les métiers de la géographie, de l'urbanisme, de l'art et du développement local et touristique de multiples façons. Tout d'abord, un 
certain nombre de ces acteurs ont une formation en géographie. D'autres ont été formés aux Beaux-Arts, ont suivi des cursus de design, de graphisme, ont une formation et/ou des expériences en gestion de projets culturels. Mais surtout, au-delà de ces parcours de formation, ces nouveaux intermédiaires réinterrogent les modes et métiers de l'action territoriale, dans leurs imbrications avec des politiques culturelles et/ou d'accompagnement du renouvellement urbain. L'enjeu de cet article est alors double. Tout d'abord, il s'agit de caractériser les nouveaux métiers et compétences qui opèrent ce lien entre street art et espaces urbains, et qui contribuent ainsi à une territorialisation du street art, y compris dans ses liens aux publics et habitants. Le second enjeu concerne la façon dont ces métiers d'intermédiation, opérant entre institutions publiques, secteur privé, habitants et artistes, transforment les modalités et les finalités des interventions (artistiques) en ville.

5 Ces paroles ont été collectées durant une journée organisée en $2017^{1}$, consacrée à la valorisation du street art, et plus spécifiquement une table-ronde impliquant différents acteurs de la valorisation du street art, illustrant différentes modalités du système d'intermédiation existant entre street-artist et contexte urbain d'implantation des œuvres. Ces propos retranscrits constituent le matériel de départ de cette synthèse qui s'efforce de rester au plus près du dit des acteurs.

\section{Une diversité d'acteurs et d'objectifs à partir du street art}

6 La journée a permis de donner la parole à tout un ensemble d'acteurs dont les compétences et les pratiques se situent aux frontières de l'art et de la ville. Ces acteurs travaillent seuls ou en associations, ils se positionnent sur une gamme étendue de profils, de l'artiste intervenant voire organisant des balades à des intermédiaires du street art, accompagnant des projets en ville, depuis leur production jusqu'à leur valorisation. Ces présentations font alors apparaître une diversité de métiers conduisant à l'association street art/espaces urbains, relevant d'une pluralité d'objectifs donnés aux interventions artistiques.

7 Elles témoignent en outre de l'évolution de ces métiers pourtant récents, en lien avec la diversification du statut des commanditaires des projets: élus locaux, institutions culturelles et touristiques, professionnels de l'urbanisme et l'aménagement, et acteurs privés.

8 - JBC. Je suis artiste, depuis maintenant quelques années. J'ai vécu les choses de l'intérieur d'une certaine manière. J'ai commencé comme pas mal de monde par des interventions dans la rue, et peu à peu j'ai été sollicité par les différents acteurs du street art: par des associations, par des acteurs informels, puis par les collectivités territoriales, et la nature même de mon travail a évolué sous l'effet des sollicitations.

9 Les autres acteurs de cette table ronde constituent des figures de ces intermédiaires, allant des structures facilitant l'intervention artistique en ville, par la gestion de murs par exemple, à des acteurs spécialisés dans l'accompagnement de l'œuvre en relation avec des institutions.

10 - Elise Herszkowicz (EH). J'ai monté une association il y a 6 ans, Art Azoï, qui est devenue avec les années opérateur culturel pour la mairie du XXe arrondissement en matière d'art urbain, pas que du $\mathrm{XX}^{\mathrm{e}}$ mais c'est le territoire dans lequel on intervient le 
plus, et c'est un partenaire privilégié pour nous. J'ai d'abord monté cette association à partir d'un projet de mur à programmation, rue des Pyrénées, qui a la particularité d'être très long : 40 mètres de long.

11 - Olivier Landes (OL). Je suis géographe de formation à la base, et j'ai ensuite évolué vers l'urbanisme, puis j'ai créé l'association Art en Ville, dont l'objet est de mettre en relation les artistes avec les acteurs institutionnels de la ville.

12 - François Roblot (FR). Je suis en charge du développement au Comité Départemental du Tourisme (CDT) du Val-de-Marne, géographe également de formation. Ce CDT est en charge du développement du tourisme de ce département en banlieue, pas toujours facile à présenter et promouvoir y compris auprès des élus ou acteurs du territoire. Notre structure est assez récente par rapport aux autres CDT en France, elle a été créée en 2000

13 - Damien Cacouault (DC). Je représente deux structures ici, à la fois mon métier qui est plus sur le côté développement territorial, car je travaille pour l'association Campus Urbain, concernée par tous les aspects du développement territorial du développement économique, culturel, sociétal aux enjeux de l'enseignement supérieur. Je suis aussi trésorier de l'association Vitry'n urbaine, association de valorisation de l'art urbain.

14 Ces différentes présentations mettent l'accent sur les nouvelles médiations opérées autour du street art, comme pour Vitry'n urbaine.

15 - DC. Le phénomène street art est repris par de nombreux acteurs, les pouvoirs publics mais aussi les bailleurs sociaux qui commencent à avoir un rôle important dans la promotion et dans l'habillage des bâtiments, en interne et en externe par le recours à des artistes. Dans le Val-de-Marne, il n'y a pas beaucoup de structures qui accompagnent tous les programmes, toutes les envies d'art pour ces bâtiments. Donc on est sollicité régulièrement, par des bailleurs sociaux, des centres sociaux, des mairies, ou des acteurs privés. Vitry'n urbaine est identifié comme étant un interlocuteur pouvant mettre en relation une façade, un projet et un artiste.

16 Les positionnements dans le spectre de ces médiations varient alors, d'acteurs qui définissent leurs activités avant tout en lien avec le street art de façon large à des acteurs pour lesquels le street art constitue un moyen au service d'enjeux de développement local ou touristique plus large.

17 Ainsi pour Art Azoï les objectifs relèvent de la promotion artistique (sans reprendre nécessairement comme entrée exclusive le street art) et du rapport des usagers à leur ville.

18 - EH. Au sein de l'association nous avons une préoccupation assez forte, qui est de rendre ses lettres de noblesse au graffiti, c'est la base pour nous de cette culture, et d'essayer de faire en sorte que les artistes puissent avoir des lieux d'expression qui soient aussi des lieux qui soient pas forcément destinés à peindre. [...] Je ne suis pas urbaniste. Ma préoccupation c'est plutôt vivre ma ville : ce qui m'intéresse c'est qu'on soit acteur de notre ville, qu'on se la réapproprie, en y installant de l'art, en diffusant de l'art plutôt que de la pub. En tous cas pour nous s'inscrire dans le territoire, c'est plus mettre en avant les interactions qu'on peut avoir avec les habitants, les propositions artistiques qu'on nous formule.

19 D'autres acteurs se définissent davantage en lien avec des objectifs de développement territorial. C'est le cas du CDT du Val de Marne pour lequel le street art constitue l'un 
des opérateurs de la diversification voire de différenciation du tourisme et de l'image du territoire.

20 - FR. Durant les premières années de fonctionnement du CDT nous avons plutôt mis en avant les bords de Marne, les guinguettes, correspondant à l'idée du territoire que se faisaient à l'époque les touristes, visiteurs et un peu les habitants. Puis à partir de 2012 avec le schéma départemental du tourisme 2013-2018, nous avons souhaité travailler sur de nouveaux territoires, sur de nouvelles offres, pour donner un nouveau regard sur le département et peut-être quitter un peu une image nostalgique des bords de Marne. Donc nous sommes allés arpenter ces territoires de banlieue, rencontrer des collectivités et des associations. Nous sommes allés découvrir Vitry et là nous avons eu un choc... Nous nous sommes dit qu'il y avait un vrai potentiel et nous avons commencé à travailler sur le street art avec les acteurs locaux qui n'étaient pas encore structurés. Nous les avons accompagnés pour faire connaître cet art au public local et francilien.

21 La création de Vitry'n urbaine répond à un même souci de développement et de changement de l'image du territoire.

- DC. L'origine de la création de Vitry'n urbaine, c'est l'image négative de Vitry, avec cette connotation ville de banlieue, avec le périph' qui crée des barrières psychologiques assez dures. À l'inverse, partant de la présence de nombreux artistes et d'œuvres sur le territoire, l'idée était de proposer des balades urbaines, justement pour donner à voir et valoriser ce qui s'y passe, et montrer que Vitry c'est une ville d'art, avec des artistes qui viennent du monde entier pour peindre à Vitry. Donc à travers les balades urbaines, l'objectif c'était de faire venir les gens à Vitry, dans une logique de promotion du territoire et de changement de regard.

\section{Le rapport au territoire}

Le rapport au territoire de ces différents acteurs varie de façon importante, entre acteurs se définissant avant tout par un territoire d'action et ceux en position d'intermédiaires agissant dans une pluralité de territoires. Plusieurs géographies se dessinent et se recoupent au fil de leurs circulations ce qui contribue à la contiguité ou à la connexité entre territoires, à la création de cohérence territoriale et nourrit les réflexions sur les rapports entre centres et périphéries. Ainsi le street art alimente des pratiques d'appropriation et de découverte de la ville, de façon explicite, à différentes échelles et selon des temporalités variées, en s'appuyant plus ou moins sur les caractéristiques locales.

24 Cela consiste d'abord en un travail localisé, dans l'espace public, en proposant des occasions et des lieux d'intervention. L'association Art Azoï gère des murs dans le $\mathrm{XX}^{\mathrm{e}}$ arrondissement, pensés comme autant d'occasions d'expression artistique, impliquant un renouvellement régulier des œuvres.

- EH. Je propose à des artistes d'intervenir très régulièrement, on change tous les deux mois. Du coup, ils peignent dans la rue, et sont en prise directe avec la rue, comme ils le font de manière illégale aussi par ailleurs. Mais là ils ont le temps de peindre, d'échanger avec les gens, et ainsi les gens peuvent avoir accès à une programmation qui change régulièrement. Au fur et à mesure, plusieurs murs à programmation sont entrés dans le projet de l'association, trois à présent (mi 2017). 
26 Cela s'inscrit en prolongement d'autres initiatives en faveur du développement artistique, montrant une double action favorisant le temps de la création et la valorisation, jouant de la circulation entre dispositifs intérieur et extérieur.

27 - EH. Auparavant je travaillais à Confluences, qui a fermé maintenant. C'était un lieu d'engagement artistique, dans le $\mathrm{XX}^{\mathrm{e}}$ arrondissement de Paris aussi, et je continue à monter des expositions et à faire en sorte que les artistes puissent avoir des résidences, avoir le temps de créer, avoir un lieu pour créer. Donc on organise des expositions, ou des interventions in situ.

28 D'autres interventions se déroulent de façon plus éclatée dans l'espace et traduisent des géographies démultipliées voire en réseau. L'association Art en Ville assure des opérations à la demande de collectivités et de professionnels de l'urbanisme et l'aménagement, en lien avec l'art urbain, dans plusieurs communes, créant des connexions entre centres et différents types de périphéries, au gré des sollicitations.

29 - OL. La première grande opération de l'association a été la mise en valeur du fort d'Aubervilliers, alors une friche en transition, anciennement une casse automobile, propriété du Grand Paris Aménagement appelée à devenir quartier ces prochaines années. La mise en valeur de ce patrimoine, du fort intérieur, a été réalisée par une cinquantaine d'artistes sur le sujet de la transition et le lieu a été ouvert durant l'été 2014. L'idée de nos projets est de toujours faire écho à la ville et finalement au lieu dans lequel les artistes interviennent, dans une démarche in situ. Une autre intervention a consisté en la mise en valeur d'une démolition à Montfermeil par l'artiste Kouka : il s'agissait d'une intervention en hommage aux voisins, au voisinage, aux amitiés et aux solidarités entre les habitants, donc c'est en fait la grue, la démolisseuse, qui a construit cette œuvre, ce qui est assez conceptuel. Puis avec l'association a été organisée la triennale de la ville de Rouen, l'été dernier, et on a accompagné la réalisation d'une quinzaine de fresques.

30 L'horizon territorial s'élargit aussi par le biais des partenariats entre collectivités (et d'autres types d'acteurs), dans le cadre du contrat de destination «Paris,la ville augmentée ", de la mise en place d'itinéraires trans-territoires à partir du street art. Le street art devient alors un produit touristique métropolitain, décliné en balades selon les quartiers.

31 - FR. Le contrat de destination a été signé entre l'État, l'OTCP (Office du Tourisme et des Congrès de Paris), les CDT de la petite couronne, l'IREST (Institut de Recherche et d'Études Supérieures du Tourisme - Université Paris 1 Panthéon-Sorbonne), la RATP, le Welcome City Lab pour répondre aux enjeux touristiques de la destination Paris, d'essayer d'enrichir l'image de la destination Paris, notamment auprès des jeunes Européens en comparaison de Berlin, Amsterdam, Londres ou Barcelone. L'enjeu est de diversifier l'image plutôt classique, romantique et concentrée sur l'hypercentre touristique de Paris pour avoir une offre complémentaire à celle qui existe aujourd'hui, à travers cinq grands axes : l'art dans la ville, la ville cosmopolite, la nature en ville, la ville festive et l'art contemporain. L'objectif de ce contrat est justement de travailler ces différents axes avec les acteurs du tourisme mais aussi différents acteurs locaux, qui concernent des territoires qui ne sont pas forcément en plein centre de Paris, mais souvent les arrondissements périphériques et les villes de banlieue proche, et qui aujourd'hui touchent un public plutôt francilien et parisien. Le street art est alors un des supports choisis pour participer à la création de cette offre complémentaire, vers de nouveaux territoires. 
Dès lors les opérations de valorisation du street art peuvent être vecteurs de nouvelles continuités territoriales, transcendant des limites administratives.

- DC. Une première balade prochainement partira de Paris XIII ${ }^{e}$ jusqu'à Vitry, en lien avec fresh street art tours, qui fera la partie XIII ${ }^{\mathrm{e}}$ puis on prendra un bus pour la partie Vitry. Un projet plus important concerne le Grand Paris Street Art, porté par Enlarge Your Paris, qui partira d'Arcueil, remontera dans le XIII', et redescendra sur Vitry, dépassant le périph' et sortant des frontières un peu classiques.

\section{La relation aux habitants et au public : de l'intermédiation à la participation?}

34 Les différents participants à la table-ronde constituent des intermédiaires, selon plusieurs directions. D'abord ils assurent la relation entre artistes, créations et institutions, mais aussi prennent en charge leur articulation avec habitants et publics. Certains acteurs jouent alors un rôle de facilitateur et d'interface. Cela suppose, de façon habituelle dans les métiers de l'aménagement, la mise en place de démarches participatives d'intégration des habitants. Il y a alors une thématisation de l'action des publics selon les différents rôles qui leur sont attribués, depuis le choix de l'œuvre, en passant par sa fabrique jusqu'à sa réception.

D'abord le public est participant, sollicité en amont de la réalisation, selon différentes modalités proches de la consultation ou de dispositifs plus participatifs, vecteurs de l'acceptabilité de l'œuvre, pensés pour permettre un ancrage facilité dans le temps long, voire pour développer de nouvelles pratiques artistiques (pratiques photographiques par les habitants par exemple). Cette nécessaire prise en compte des habitants fait consensus durant la table-ronde, qu'elle soit informelle le temps de réalisation de l'œuvre ou qu'elle fasse l'objet de projets spécifiques sous la conduite d'intermédiaires, en amont, pendant et en aval des réalisations.

6 - EH. Quand on est dans le milieu des habitations des gens, c'est important d'avoir une consultation, de prendre le temps, que l'artiste fasse des rencontres avec les habitants.

- JBC. La dimension participative, que les habitants aient leur mot à dire, est quand même importante pour une œuvre d'art, surtout si elle a une certaine taille et si elle modèle l'espace urbain. Si les gens ont une œuvre qui ne leur correspond pas et qui n'a aucun lien avec le quartier ou l'histoire du quartier, je peux comprendre que ça puisse générer de la frustration et de la défiance, et nous les artistes on ne peut pas être insensibles à ça. À partir du moment où on offre une œuvre d'art dans l'espace public et au public : il faut qu'on soit conscient aussi de ce qu'on va générer derrière.

- OL. Concernant la valorisation, ce qui est important pour l'association Art en Ville c'est vraiment d'impliquer les publics et les habitants, étant donné qu'on est sur des fresques qui vont rester dans le paysage urbain de nombreuses années, qui sont parfois massives. Inévitablement les habitants sont concertés au maximum au préalable, en amont de la peinture. Nous proposons également tout un contenu pédagogique dans les écoles et les centres sociaux des environs. L'idée est de ne pas passer à côté du formidable potentiel social contenu dans l'arrivée d'une fresque dans un quartier. Ainsi il y a une sorte de valorisation du projet, au même titre que pour un projet d'urbanisme, qu'on vient valoriser via la participation des publics. Et naturellement le temps du chantier est extrêmement valorisable. 
39 Le public est spectateur de l'œuvre en train de se faire, ce qui est rendu possible par la dimension processuelle et performative du street art, et permet un temps d'échange avec l'artiste.

40 - FR. Le fait que le street art soit justement dans la rue facilite l'échange avec le public, souvent par le contact direct avec les artistes, qui peuvent expliquer leur démarche, à l'inverse d'autres arts qui sont dans les murs. On essaie d'aller à la rencontre des artistes dans leur atelier mais aussi directement dans la rue, sur de l'événementiel. Par exemple, on organise régulièrement tous les ans une grande marche. Pour moi tout l'intérêt du street art c'est cet échange facilité avec du public, de manière organisée ou pas.

41 - JBC. La différence entre l'art urbain et un art plus traditionnel, c'est la dimension performative, c'est-à-dire que pour nous les artistes c'est un medium privilégié d'accès au public, et de valorisation du travail de l'artiste par le public. D'un côté il y a la dimension urbex : on peut faire des œuvres dans des friches éloignées d'une manière clandestine, qui se rattachent plus à la tradition du graffiti, mais ce qu'on aime de l'art urbain ou du street art c'est précisément ce contact avec le public.

42 Le public devient participant de façon accrue également lorsqu'il est impliqué dans la conception de l'œuvre et qu'il en reste une trace in fine. Là aussi des associations jouent un rôle d'intermédiaire, notamment via des projets pédagogiques.

43 - DC. Par exemple, on est en train de porter un projet dans un lycée professionnel, avec un grand mur devant le lycée, et le lycée nous a contactés pour avoir au cours de l'année un projet pédagogique impliquant les élèves. Tout un travail de réflexion a été mené avec les artistes pour aboutir in fine à la réalisation du mur. Donc c'était une coconstruction avec des discussions, de travail en temps de cours mené par les profs, et de l'interaction avec les artistes. Dans une logique similaire, un bailleur social nous a contactés pour décorer tout l'espace affecté aux boîtes aux lettres du rez-de-chaussée et le travail proposé par l'artiste était de travailler à partir des mains de tous les habitants, de les prendre en photo et de les représenter dans tout l'espace, bref d'intégrer le public dans la conception d'œuvre.

44 Le public de l'œuvre est aussi évoqué et thématisé dans le temps d'après, celui de la réception et de l'appropriation. Mais cette réception ne se construit pas seulement $a$ posteriori : elle se prépare en amont.

45 - OL. Dans le cas d'œuvres pérennes se pose plus encore la question des habitants, du public du quotidien, avec la question de savoir si réellement l'appropriation a lieu. Travailler sur la nécessaire appropriation d'un objet plastique en espace public, c'est pour moi très important pour accompagner l'arrivée d'une œuvre d'art qui va être monumentale dans l'espace public. On a diverses méthodes préalablement à son arrivée. Pour ne pas opérer un simple parachutage d'artistes pour mettre de l'art dans l'espace public, parfois sur des très grandes surfaces, on propose des méthodes plus contextualisées, de façon à ce que les gens se disent par exemple « mais oui ça traite du végétal dans la ville car on est dans un quartier avec du végétal, ça parle de nous ». C'est réellement ce qu'on essaie de faire en favorisant l'appropriation.

46 L'implication des habitants se prolonge après le temps de la production, à travers des dispositifs qui permettent de développer un nouveau regard sur la ville, et de former à des pratiques artistiques qui s'inscrivent dans la continuité du street art in situ et permettent sa médiatisation. 
47 - DC. On propose des ateliers avec des artistes, donc on essaie de rapprocher l'artiste du public et de les faire travailler ensemble pour que chacun reparte avec l'œuvre coconstruite. On organise aussi des balades ou on essaie de mélanger cours de photographie et street art: de nombreuses personnes sortent photographier du street art souvent avec la frustration de prendre des photos de face, du coup l'idée c'était de proposer le travail avec un photographe pour essayer de changer d'angle et changer de regard et changer la manière de regarder l'art dans la rue, et changer la manière de le ramener chez soi, sur les outils numériques.

\section{Valorisations touristiques : l'art et la ville}

48 Le street art alimente donc des pratiques de valorisation touristique, que ce soit au niveau des objectifs (faire découvrir de nouveaux quartiers) ou des outils mobilisés (la balade urbaine, la visite). Les acteurs du tourisme se saisissent du street art afin de diversifier les balades proposées, ou de permettre la découverte de quartiers jusque-là en retrait. D'autres acteurs se saisissent des outils ou du langage de la valorisation touristique pour assurer la médiation du street art voire pour développer d'autres pratiques artistiques (photographie). La balade urbaine constitue l'instrument privilégié par ces acteurs, notamment pour attirer de nouveaux publics franciliens ou touristiques. Le street art fonctionne alors comme point de départ pour des objectifs de valorisation in situ.

49 - FR. En termes d'outil la balade urbaine permet des échanges avec les artistes, et dans les partenariats pour des balades, avec Vitry'n urbaine ou d'autres acteurs, on privilégie des rencontres avec des habitants, des passionnés, ou des associations. Notre démarche est vraiment d'aller au plus près du terrain, avec ces acteurs qui sont les mieux placés pour parler de leur territoire.

50 Les habitants participent aussi à ces démarches de mise en découverte et balade au prisme du street art, quand certains publics locaux ne constituent pas eux-mêmes les cibles explicites des démarches menées. Le street art comme vecteur de mise en réseau d'autres pratiques et espaces artistiques, notamment parce qu'il permet de toucher des publics jeunes, familiaux...

51 - OL. En fait ces quartiers sont l'objet, ramènent du tourisme, il y a des groupes qui viennent avec des appareils photo, qui prennent des photos, c'est intéressant puisque ça brasse de nouvelles populations dans la ville. Par exemple à Rouen sur le quartier des Sapins, des habitants, quand ils reçoivent de la famille le dimanche, les amènent faire le tour du quartier. Les habitants se sont appropriés l'initiative et deviennent les nouveaux ambassadeurs de l'art urbain.

52 - DC. On organise aussi pas mal de choses avec un public plus scolaire de collège ou lycée, plutôt dans le sud parisien, et on essaie dans ce cadre de créer des interactions avec d'autres structures de Vitry, quand des structures pédagogiques nous contactent pour des balades : le Musée d'art contemporain Mac Val, des théâtres, la Briqueterie qui est un espace de danse, pour ouvrir le réseau un maximum. Le street art n'est pas la seule forme d'art sur le territoire : on essaie de créer des ponts et des interactions entre les différentes structures, que ça ne se cantonne pas à une approche. 
53 Toutefois peut être assumée aussi une non-valorisation explicite, ce qui ne signifie pas une non-réflexion sur la place des publics mais se rapproche de la dimension non encadrée du street art.

54 - EH. Il peut y avoir des instits qui viennent avec la classe, donc on prévient les artistes que des gens vont passer ... Il y a des moments plus organisés que d'autres mais ce qui nous intéresse c'est ce côté justement informel, spontané et pas organisé des gens qui s'arrêtent, s'approprient cette proposition qui est faite et qui change... C'est un peu paradoxal ce monde qui est en même temps secret, qui avance un peu masqué, et en même temps peut être vu de tout le monde. Mon inclinaison ne va pas vers l'organisation de visites guidées, parce que ça m'intéresse plus d'échanger là-dessus comme ça au gré du passage des gens ...

55 Se pose aussi la question de ce qui est valorisé : street art et ville se renforcent mutuellement, permettant un nouveau regard sur la ville ou la découverte de nouveaux quartiers.

56 - DC. On part du principe que la balade urbaine est un outil de valorisation, mais que l'art est aussi un prétexte pour faire venir les gens, discuter, échanger. L'idée c'est que les gens viennent pour l'art, mais une fois sur place, ce qui est intéressant, c'est comment l'art s'inscrit dans la ville et comment cela interagit avec les habitants, comment le public interagit. L'art constitue l'appel, et après quelque chose de plus global peut être valorisé : la diversité culturelle, l'architecture, etc.

57 - OL. On voit vraiment l'aspect hybride entre ville et art, art urbain, street art. Chez Art en Ville on souhaite mettre en valeur la médiation autour de ce qui se fait car à la fois c'est de la médiation artistique mais cela peut aussi être de la médiation urbaine. Via l'artiste on va révéler un pignon un peu moche, une arrière-cour, une porte, un hangar, et en fait on peut aussi raconter l'histoire de cette porte, de ce hangar ou de ce pignon ou de ce quartier via les interventions artistiques ou au moins donner un nouvel œil pour ouvrir un nouveau regard vers des zones qui sont pas forcément attractives.

58 Ces démarches de valorisation constituent-elles alors des démarches de niche, pour un public spécifique ? Les intervenants soulignent plutôt la diversité des publics et de leurs motivations.

59 - DC. Sur les publics, sur les balades qu'on organise en partenariat avec le Comité du tourisme c'est plutôt un public francilien et de tout âge, vraiment c'est assez étonnant. Il y a aussi pas mal de jeunes qui s'implantent à Vitry, plutôt trentenaires, et font ces balades pour comprendre leur ville. Quand on avait organisé les balades sur les ateliers photo, des gens venaient de beaucoup plus loin, Orléans ou Reims.

60 - OL. On est en train de percevoir que le street art n'existe pas, c'est plutôt des ou les street arts, pour ne pas dire le graffiti, la pratique sauvage, le collage, le pochoir, le muralisme. Finalement ce mot englobe une innombrable réalité et ce qui se passe à Vitry, dans le XIX', ce qu'on fait nous à Art en Ville, on fait partie d'une grande partie d'intervenants artistiques dans la rue auxquels il faudrait d'ailleurs ajouter les gens qui font de la sculpture, de l'installation. [...] Le rapport au public est radicalement différent si tu fais une grande fresque murale qui va rester là 20 ans ou si tu fais un one shot dans une courette avec un pochoir qui va faire des tonnes de vues sur les réseaux sociaux et qui va être vu par 8 personnes au quotidien. C'est là que le rapport au public est très différent parce que la pratique est très différente entre une démarche très accompagnée, institutionnelle, rémunérée, gratuite, humoristique, ou décalée. Il y a 
même des artistes qui travaillent pour des marques, qui font carrément de la publicité ... Les publics sont vraiment différents.

61 Cette diversité des publics a alors des incidences sur les façons de faire, comme l'explique Thom Thom, artiste urbain, organisateur de balades street art $^{2}$.

Les gens qui font des balades, avant tout, ils veulent faire des balades. Ça il ne faut pas l'oublier pour les offres touristiques. Les gens qui viennent des grandes couronnes franciliennes, d'assez loin, ce qu'ils voulaient c'est marcher pendant trois heures, après, que je leur montrais des choses ça leur plaisait mais ce qu'ils prenaient plaisir à faire surtout, au-delà de quelques photographes, c'était se réapproprier le territoire à pied. C'est pour ça que j'ai mis en place en place une balade qui part de Bobigny, puis qui suit tout le canal de l'Ourcq pour revenir ensuite dans le XIX' arrondissement de Paris.

Toutefois Thom Thom souligne aussi la spécificité de la balade comme outil de découverte de l'art de rue, de par la dimension éphémère et contextuelle de cet art.

- TT. Là les gens sont ravis, dans cet environnement justement de graffiti sauvage ... et je leur dis : prenez des photos, vivez le et revenez dans cinq ans parce que tout le long de Pantin ça va changer énormément et c'est ça qui est intéressant avec le graffiti pur et dur, c'est que c'est un marqueur de l'évolution de l'urbanisme. Un graffiti ne peut pas vivre des décennies et des décennies: vous voyez le graffiti ça veut dire que l'entrepôt va être détruit ... Les personnes connaissent vaguement le street art, ils s'intéressent un peu, ce qui les intéresse c'est surtout de découvrir la ville d'une manière différente. Mais durant ces balades urbaines on va voir des œuvres plus contextuelles, ce ne sont pas des choses d'Instagram, ce sont des œuvres nonphotographiables qui font sens parce qu'elles sont là. Par exemple un artiste, $\mathrm{Jim}^{3}$, pose des pansements sur les fissures, en photographie ça ne passe pas, ce n'est pas aussi intéressant que le voir; un autre artiste, Milo Project, qui pose des sorties de secours dans des endroits complètement incongrus ${ }^{4}$, pareil une photo ça n'a pas de sens mais de se balader et voir ça dans la rue ...

\section{Ce que ces nouvelles pratiques et intermédiations font au street art?}

65 Ces pratiques de territorialisation et d'intermédiation autour du street art contribuent aussi à faire évoluer le street art lui-même, dans ses contenus, ses temporalités et ses modes de faire. Se pose tout d'abord la question des effets de l'institutionnalisation ou de la commande sur les pratiques.

- JBC. Au départ les formes artistiques que j'avais choisies dans l'espace urbain étaient spontanées, avec un caractère plus politique, et finalement cette espèce de marotte que j'avais d'aller dans les rues, de faire un petit collage, c'est un temps où j'avais un peu plus de temps libre qu'aujourd'hui car je débutais ma démarche... Avec le temps, et par l'effet par exemple des associations qui commencent à nous solliciter pour des festivals, on a moins de temps pour intervenir de manière spontanée dans l'espace public, et c'est la nature même de l'œuvre d'art qui change: on nous demande d'être plus professionnel, de respecter les temps impartis, de peindre sur des surfaces plus grandes, de respecter des thématiques aussi... J'ai un certain goût pour des thèmes parfois un peu provocateurs, un peu politiques, borderline; je sais très bien que si je travaille pour une collectivité locale je vais être un peu plus prudent sur les sujets que 
je vais aborder, tant au niveau de la forme, des thématiques, que des médiums utilisés, parce que on nous demande aussi de la pérennité parfois (le collage ne fonctionne plus à ce moment-là faut faire de la peinture). Il y a une évolution du travail qui se fait et pour nous ce sont des portes qui s'ouvrent, des champs des possibles qu'on n'avait pas distingués avant et qui font évoluer notre pratique artistique. Après ça peut aussi conduire à une standardisation des formes et des genres artistiques qu'on propose et on peut se laisser piéger effectivement. Le piège serait de se cantonner à un art qui plaise au plus grand nombre et laisser de côté un aspect un peu plus spontané ou un peu plus provocateur, un peu plus citoyen.

67 - EH. Est-ce que les artistes se censurent ou pas, est-ce que le fait que ce soit devenu tellement à la mode influe sur leur travail ? Par exemple il y a deux ans on a fait intervenir quinze artistes sur les vitrines du ministère de la culture et en tant que commissaire, le préalable était qu'on ne demandera pas de maquette aux artistes. Pour moi comme pour les murs à programmation à partir du moment où je propose un artiste, je ne demande pas de maquette. Les artistes font de l'art contextuel, c'est une des particularités de l'art urbain, d'interagir avec le lieu dans lequel ils s'expriment. Ils sont imprégnés de ce qui se passe autour d'eux, de ce qu'ils en voient. En revanche pour les murs pérennes on ne peut pas échapper à la maquette et à une validation institutionnelle, à travers la procédure de DP (déclaration préalable), auprès de la direction de l'urbanisme, qui elle-même saisit les architectes des bâtiments de France à proximité d'un monument historique, et aboutissant à donner un avis sur la couleur, la forme, avec un délai d'instruction. Donc là il y a une espèce de censure institutionnelle, c'est le principe de la commande pour laquelle il faut fournir une maquette assez précise.

68 - OL. Ça dépend aussi des institutions. À Rouen on a eu quinze fresques dans la ville, dont sept qualifiées de semi-pérennes, sans maquettes, donc on a eu aussi la chance de travailler avec une institution assez libérale dans son genre.

\section{Conclusion}

69 Tout un ensemble de métiers et compétences assurent l'articulation entre street art et territoires urbains, du point de vue de l'intégration à des projets de territoire, de la médiation entre artistes et collectivités ou institutions publiques, de l'accompagnement de démarches participatives associées à la réalisation d'œuvres dans l'espace public, et de la promotion et valorisation touristiques de territoires métropolitains au centre et en périphérie.

70 Ces métiers sont récents et les compétences évoluent déjà au fil des collaborations et des circulations de chacun.

71 Deux lectures peuvent alors être proposées. Ces métiers et compétences s'inscrivent en continuité des modalités de l'intervention en ville: intermédiation, participation, valorisation auprès de publics variés. À ce titre, le street art peut apparaître comme une thématique nouvelle. Toutefois, on peut aussi y lire l'émergence d'une modalité renouvelée de la fabrique et valorisation urbaine, se traduisant par une territorialisation explicite des démarches artistiques, qui aurait pour effet la création de nouveaux réseaux entre territoires valorisés et entre acteurs, tout en agissant sur les formes du street art. 


\section{NOTES}

1. Journée d'étude organisée le 5 mai 2017 par Pauline Guinard, Sébastien Jacquot et Clotilde Kullmann, intitulée "Les valorisations territoriales et touristiques du street art ", à Paris (Grands Voisins, XIVe), avec le soutien de l'ENS, de la Fondation de la maison des sciences de l'homme et de l'EA EIREST (Université Paris 1 Panthéon-Sorbonne).

2. Présent parmi le public de cette table ronde.

3. https://www.urbacolors.com/fr/artist/jim

4. https://www.instagram.com/p/Bj4fhVllhof/?taken-by=milo.project

\section{AUTHORS}

\section{SÉBASTIEN JACQUOT}

Sébastien Jacquot, Sebastien.Jacquot@univ-paris1.fr, est maître de conférences en géographie à

l'Université Paris 1 Panthéon-Sorbonne. Il était le modérateur de la table ronde.

\section{OLIVIER LANDES}

olivier Landes est directeur de l'association Art en Ville.

\section{ELISE HERSZKOWICZ}

Elise Herszkowicz est directrice de l'association Art Azoï.

JBC

$\mathrm{JBC}$ est artiste.

FRANÇOIS ROBLOT

François Roblot est responsable du Service Développement, Comité Départemental du Tourisme 94.

\section{DAMIEN CACOUAULT}

Damien Cacouault est membre de l'association Vitry'n urbaine

\section{THOM THOM}

Thom Thom est artiste urbain. Présent dans la salle, il intervient dans le débat au sujet des balades. 